\title{
ULTRAVIOLET EMISSION FROM STELLAR POPULATIONS WITHIN TIDAL TAILS: CATCHING THE YOUNGEST GALAXIES IN FORMATION?
}

\author{
S. G. Neff, ${ }^{1}$ D. A. Thilker, ${ }^{2}$ M. Seibert,${ }^{3}$ A. Gil de Paz,${ }^{4}$ L. Bianchi, ${ }^{2}$ D. Schiminovich,${ }^{3,5}$ D. C. Martin, ${ }^{3}$ \\ B. F. Madore, ${ }^{4}$ R. M. Rich, ${ }^{6}$ T. A. Barlow, ${ }^{3}$ Y.-I. Byun, ${ }^{7}$ J. Donas,${ }^{8}$ K. Forster, ${ }^{3}$ P. G. Friedman, ${ }^{3}$ \\ T. M. Heckman, ${ }^{9}$ P. N. Jelinsky, ${ }^{10}$ Y.-W. Lee, ${ }^{7}$ R. F. Malina, ${ }^{8}$ B. Milliard,${ }^{8}$ P. Morrissey, ${ }^{3}$ \\ O. H. W. Siegmund, ${ }^{10}$ T. Small, ${ }^{3}$ A. S. Szalay, ${ }^{9}$ B. Y. Welsh, ${ }^{10}$ and T. K. Wyder ${ }^{3}$ \\ Received 2004 July 9; accepted 2004 September 27; published 2005 January 17
}

\begin{abstract}
New Galaxy Evolution Explorer (GALEX) observations have detected significant far-UV (FUV; $1530 \AA$ ) and near-UV (NUV; $2310 \AA$ ) emission from stellar substructures within the tidal tails of four ongoing galaxy mergers. The UV-bright regions are optically faint and are coincident with $\mathrm{H}$ I density enhancements. FUV emission is detected at any location where the $\mathrm{H}$ I surface density exceeds $\sim 2 M_{\odot} \mathrm{pc}^{-2}$, and it is often detected in the absence of visible wavelength emission. UV luminosities of the brighter regions of the tidal tails imply masses of $10^{6}$ to $\sim 10^{9} M_{\odot}$ in young stars in the tails, and $\mathrm{H}$ I luminosities imply similar $\mathrm{H}$ I masses. UV-optical colors of the tidal tails indicate stellar populations as young as a few megayears, and in all cases ages under 400 Myr. Most of the young stars in the tails formed in single bursts, rather than resulting from continuous star formation, and they formed in situ as the tails evolved. Star formation appears to be older near the parent galaxies and younger at increasing distances from the parent galaxy. This could be because the star formation occurs progressively along the tails, or because the star formation has been inhibited near the galaxy/tail interface. The youngest stellar concentrations, usually near the ends of long tidal tails, have masses comparable to confirmed tidal dwarf galaxies and may be newly forming galaxies undergoing their first burst of star formation.
\end{abstract}

Subject headings: galaxies: evolution — galaxies: individual (Arp 295, NGC 520, NGC 5719, NGC 7771) — galaxies: interactions

\section{INTRODUCTION}

Galaxy evolution is strongly influenced by interactions and mergers. Dramatic structural changes often result from such events. Interactions and mergers occur more frequently at higher redshifts and were even more important in the early universe than today.

Tidal tails may be ejected more than $100 \mathrm{kpc}$ during strong interactions, with more than half of the neutral gas in each galaxy ending up in the tail (Braine et al. 2001). The tails are often blue (Schombert et al. 1990), and occasionally, $\mathrm{H} \alpha$ emission and young stars (a few hundred megayears) are detected, indicative of active star formation (Hibbard \& van Gorkom 1996; Knierman et al. 2003). Models predict that gas condensations in tidal tails will self-gravitate into new "tidal dwarf galaxies" (TDGs; Barnes \& Hernquist 1992; Mihos \& Hernquist 1994, 1996); such predictions are confirmed by observation (Mirabel et al. 1992; Duc \& Mirabel 1998; Braine et al. 2001). Recent work on tidal tails (Saviane et al. 2004;

\footnotetext{
${ }^{1}$ Laboratory for Astronomy and Solar Physics, NASA Goddard Space Flight Center, Greenbelt, MD 20771; susan.g.neff@nasa.gov.

${ }^{2}$ Center for Astrophysical Sciences, Johns Hopkins University, 3400 North Charles Street, Baltimore, MD 21218.

${ }^{3}$ California Institute of Technology, MC 405-47, 1200 East California Boulevard, Pasadena, CA 91125.

${ }^{4}$ Observatories of the Carnegie Institution of Washington, 813 Santa Barbara Street, Pasadena, CA 91101.

${ }^{5}$ Department of Astronomy, Columbia University, New York, NY 10027.

${ }^{6}$ Department of Physics and Astronomy, University of California at Los Angeles, Los Angeles, CA 90095.

${ }^{7}$ Center for Space Astrophysics, Yonsei University, Seoul 120-749, Korea.

${ }^{8}$ Laboratoire d'Astrophysique de Marseille, BP 8, Traverse du Siphon, 13376 Marseille Cedex 12, France.

${ }^{9}$ Department of Physics and Astronomy, Johns Hopkins University, Homewood Campus, Baltimore, MD 21218.

${ }^{10}$ Space Sciences Laboratory, University of California at Berkeley, 601 Campbell Hall, Berkeley, CA 94720.
}

Knierman et al. 2003; de Grijs et al. 2003) has established that young star clusters and associations (10-500 Myr) can form in tidal tails.

In this Letter, we present an analysis of strong UV emission associated with tidal tails in four major galaxy interactions. Hibbard et al. (2005) also report the Galaxy Evolution Explorer $(G A L E X)$ detection of in situ star formation within the tidal tails of the Antennae (NGC 4038/39).

\section{TARGETS, OBSERVATIONS, AND DATA ANALYSIS}

\subsection{Targets}

We present GALEX UV imagery (Figs. $1 a-1 d$ ) plus archival visible-band and $\mathrm{H}$ I observations (Figs. 1e-1h) for NGC 7769/ 71, NGC 5713/19, Arp 295, and the NGC 520 (Arp 157) system. They represent a range of merger stages, from very early (NGC 7769/71 and NGC 5713/19) through early (Arp 295) to nearly merged (NGC 520).

The NGC $7769 / 71$ pair $(D=56 \mathrm{Mpc})$ dominate a loose galaxy group embedded in a common $\mathrm{H}$ I envelope (Nordgren et al. 1997). NGC 7771 is part of a triplet of galaxies including NGC 7770 and NGC 7771a. A ring of higher column density encircles the NGC 7771 triplet, and lower column density H I extends in a tail ( $\gtrsim 100 \mathrm{kpc}$ to the south). Nordgren et al. (1997) suggest that the galaxies have passed perigalacticon once in a prograde (NGC 7771)-retrograde (NGC 7769) interaction in which a significant tidal tail was ejected from NGC 7771, but very little matter was removed from NGC 7769.

NGC 5713/19 $(D=25 \mathrm{Mpc})$ are a pair of interacting galaxies that form part of the larger galaxy group CfA 139 (Haynes \& Giovanelli 1991). Both galaxies have extensive H I envelopes and tidal tails (Langston \& Teuben 2001). Hibbard et al. (2001) classify this system as a prograde-prograde interaction, in which two tidal tails have been ejected from gas-rich galaxies. H I 

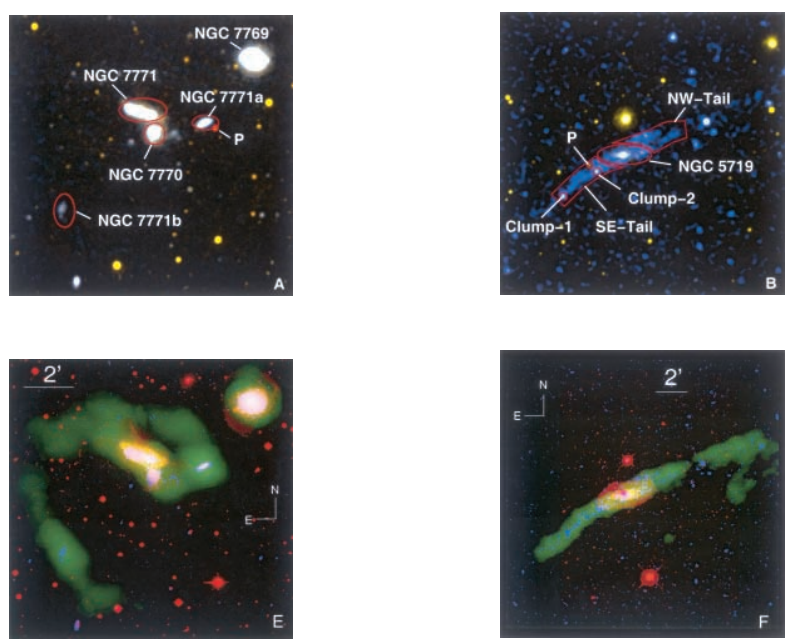

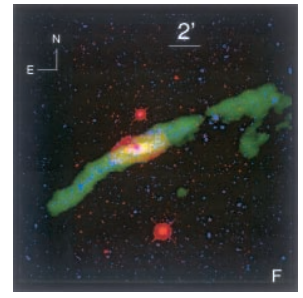

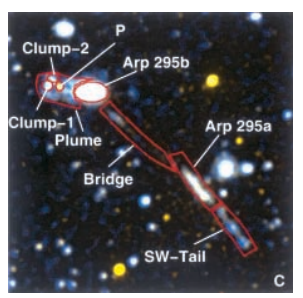
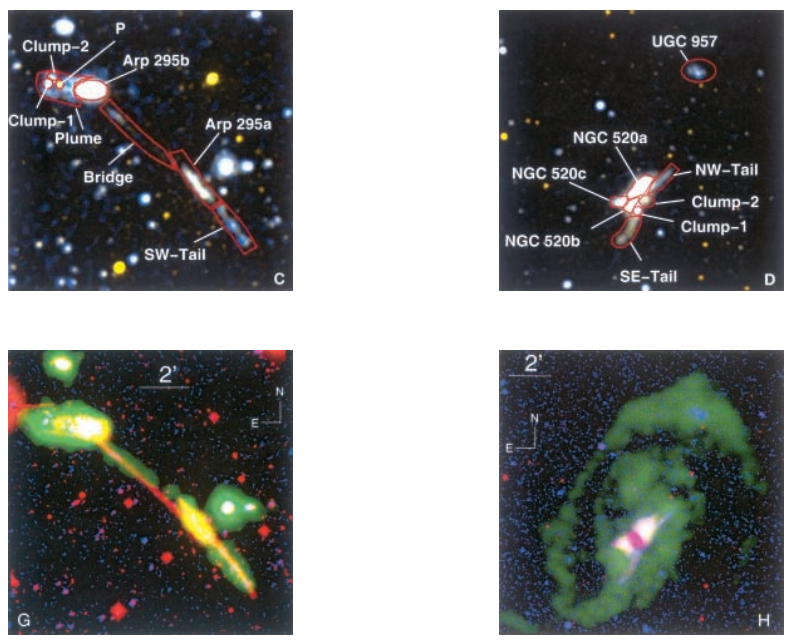

FIG. 1.-Left to right: Tidal tails associated with NGC 7771, NGC 5719, Arp 295, and NGC 520. Top panels show FUV (blue), NUV (orange), and indicate the regions used for photometry. Foreground stars are labeled "P" ("point") and were excluded from the photometry. Bottom panels show the same galaxy systems in FUV emission (blue), $R$ or $r$ band (red), and $\mathrm{H}$ I (green).

column densities are quite high in the tails $\left[(4-8) \times 10^{20} \mathrm{~cm}^{-2}\right]$. We focus on NGC 5719's tidal tail, which extends $240 \mathrm{kpc}$.

The galaxies in the Arp 295 pair $(D=69 \mathrm{Mpc})$ are the largest spirals in a loose group of galaxies. They appear connected by a dramatic optical and $\mathrm{H}$ I bridge. A luminous tail extends $\gtrsim 50 \mathrm{kpc}$ to the southwest of Arp 295a, and a plume extends $\sim 35 \mathrm{kpc}$ to east of Arp 295b (Hibbard \& van Gorkom 1996). The system apparently resulted from a prograde (Arp 295a)-retrograde (Arp 295b) encounter when an extensive tail was ejected from Arp 295a (it wraps around to appear as the bridge between the galaxies before extending off to the southwest) and when the gas-rich plume east of Arp 295b was removed but remained near the parent galaxy.

The NGC 520 system $(D=23 \mathrm{Mpc})$ is more evolved, with the progenitor disks almost merged; $K$-band peaks indicate two nuclei separated by only $\sim 5 \mathrm{kpc}$ (Stanford \& Balcells 1990; Kotilainen et al. 2001). An optical and $\mathrm{H}$ I tidal tail extends $\sim 25$ $\mathrm{kpc}$ to the south from the nuclei, then abruptly bends into a faint red plume and $\mathrm{H}$ I arm extending east and then north. These structures are embedded in a disklike $\mathrm{H}$ I distribution (Hibbard et al. 2000) that extends northward to the dwarf galaxy UGC 957 (>50 kpc separation) and then continues around to the west and south. The NGC 520 system probably resulted from a prograde-retrograde (or polar) interaction between two large disks (Stanford \& Balcells 1990). Hibbard et al. (2000) suggest that UGC 957 may be a tidal dwarf galaxy formed in the interaction.

\subsection{UV Observations}

GALEX observed each system for a single orbit in both farUV (FUV; $1530 \AA$ ) and near-UV (NUV; $2310 \AA$ ) bands. Routine data processing and calibration was as described in Martin et al. (2005). Figure 1 shows the GALEX images compared to visible and $21 \mathrm{~cm} \mathrm{H} \mathrm{I} \mathrm{imagery.} \mathrm{UV} \mathrm{emission} \mathrm{is} \mathrm{seen} \mathrm{to} \mathrm{be}$ associated with all of the interacting galaxies and their tidal tails, extending large distances $(10-100 \mathrm{kpc})$ beyond the optical extent. The images were adaptively smoothed, and morphologically distinct regions were selected for photometry (FUV images were used to define apertures at all wavelengths; Fig. 1, top). Aperture photometry was performed using custom IDL routines; sky was determined as the mean of 8-12 boxes around the target. Background-subtracted $\mathrm{AB}$ magnitudes, photometric errors, and FUV - NUV colors were then determined (Table 1) from the original unsmoothed data.
The NGC 7769/71 GALEX image (1663 s; Fig. 1a) shows strong UV features associated with both the principal galaxies and their satellites, and with a peak in the southwest $\mathrm{H}$ I tail of NGC 7771 at NGC 7771b. A faint UV halo extends west of NGC 7771 toward NGC 7770 and NGC 7771a, in agreement with the extent of the H I. NGC 7771b has the most extreme FUV - NUV value in our galaxy sample.

The disks of NGC 5719 and NGC 5713 (not shown) are easily detected in the short GALEX observation (844 s; Fig. 1b). UV emission extends well beyond NGC 5719's optical disk, continuing nearly the entire length of NGC 5719's H I tidal tail ( $240 \mathrm{kpc}$ from the nucleus). The tail FUV emission consists of very blue clumps at the locations of $\mathrm{H}$ I peaks, in addition to a more diffuse component. Very faint bridge UV emission is also detected coincident with the $\mathrm{H}$ I northwest of NGC 5719, extending toward NGC 5713.

UV emission is detected from several components of the Arp 295 system (1234 s, Fig. 1c). Arp 295b is a bright, compact source, while Arp 295a appears clumpy. UV emission is detected from Arp 295a's tidal tail and from the bridge; however, the tidal tail does not extend as far to the southwest in the UV as it does at visible wavelengths, and the UV bridge fades in the middle. The plume east of Arp 295b is conspicuous in the UV, with clump 1 (embedded in the plume) being the bluest UV location in the Arp 295 system.

The UV observations of NGC 520 (1730 s, Fig. 1d) show that the central region appears similar at UV and visible wavelengths (Hibbard et al. 2000), with FUV emission detected from the remnant galaxy bodies. The inner regions of two clumpy tidal tails are clearly visible in the UV images, extending southeast and northwest. UGC 957, the likely TDG, shows up very clearly in the FUV image and has the bluest UV colors in the system. The red plume extending east and north to UGC 957 is not detected in the UV.

\subsection{H I Observations}

$\mathrm{H}$ I maps of each interacting system were obtained using the NRAO VLA. ${ }^{11}$ Details of the array configuration, correlator setup, imaging parameters, and subsequent deconvolution are in the original papers (NGC 7769/71, Nordgren et al. (1997); NGC

\footnotetext{
${ }^{11}$ The National Radio Astronomy Observatory is a facility of the National Science Foundation d under cooperative agreement by Associated Universities, Inc.
} 
TABLE 1

Observed and Derived Properties of Regions

\begin{tabular}{|c|c|c|c|c|c|c|c|c|}
\hline \multirow[b]{2}{*}{ SySTEM } & \multirow[b]{2}{*}{ NAME } & \multicolumn{3}{|c|}{ Before Galactic Foreground Correction } & \multirow{2}{*}{$\underset{(y r)}{\log a g e^{b}}$} & \multirow{2}{*}{$\begin{array}{c}\log M_{\text {stars }}{ }^{c} \\
\left(M_{\odot}\right)\end{array}$} & \multirow{2}{*}{$\begin{array}{c}\log M_{\mathrm{H}_{1}}^{\mathrm{d}} \\
\left(M_{\odot}\right)\end{array}$} & \multirow{2}{*}{$\begin{array}{c}\sum_{\mathrm{H}_{1} \mathrm{~d}}^{-2} \\
\left(M_{\odot} \mathrm{pc}^{-2}\right)\end{array}$} \\
\hline & & FUV & FUV - NUV & $\mathrm{NUV}-r^{\mathrm{a}}$ & & & & \\
\hline \multirow{4}{*}{ NGC $7769 / 71 \ldots \ldots}$. & NGC 7770 & $16.44 \pm 0.10$ & $0.26 \pm 0.13$ & $2.86 \pm 0.09$ & $8.2 \pm 0.2$ & $9.4 \pm 0.1$ & 8.8 [7.5] & $2.7[0.1]$ \\
\hline & NGC 7771 & $16.90 \pm 0.05$ & $0.78 \pm 0.08$ & $4.46 \pm 0.06$ & $8.6 \pm 0.1$ & $10.4 \pm 0.1$ & $9.3[7.8]$ & $3.7[0.1]$ \\
\hline & NGC 7771a & $18.38 \pm 0.07$ & $0.12 \pm 0.10$ & $2.59 \pm 0.07$ & $8.0_{-0.4}^{+0.1}$ & $8.4 \pm 0.1$ & $8.8[7.4]$ & $3.6[0.1]$ \\
\hline & NGC $7771 \mathrm{~b}$ & $19.81 \pm 0.26$ & $-0.32 \pm 0.40$ & $2.43 \pm 0.36$ & $6.4-7.0$ & $7.6_{-0.4}^{+0.1}$ & $8.7[7.6]$ & $1.6[0.1]$ \\
\hline \multirow[t]{5}{*}{ NGC 5713/19 ....... } & Disk & $17.96 \pm 0.08$ & $0.89 \pm 0.09$ & $5.44 \pm 0.04$ & $8.5 \pm 0.1$ & $10.0 \pm 0.1$ & $9.1[7.8]$ & $10.1[0.5]$ \\
\hline & Southeast tail & $18.03 \pm 0.11$ & $-0.03 \pm 0.14$ & $3.27 \pm 0.09$ & $6.3_{-0.2}^{+0.5}$ & $8.2_{-0.3}^{+0.1}$ & $9.0[7.9]$ & $5.8[0.4]$ \\
\hline & Northwest tail & $18.47 \pm 0.15$ & $0.25 \pm 0.18$ & $3.68 \pm 0.10$ & $8.0_{-1.5}^{+0.3}$ & $8.5_{-0.2}^{+0.1}$ & $8.9[7.9]$ & $5.0[0.5]$ \\
\hline & Clump 1 & $20.11 \pm 0.19$ & $-0.25 \pm 0.25$ & $1.34 \pm 0.22$ & $6.4_{-0.2}^{+0.4}$ & $6.0_{-0.4}^{+0.2}$ & $7.5[6.6]$ & $4.5[0.5]$ \\
\hline & Clump 2 & $20.07 \pm 0.18$ & $-0.24 \pm 0.23$ & $2.31 \pm 0.16$ & $6.4-6.6$ & $6.8_{-0.3}^{+0.1}$ & $7.8[6.6]$ & $8.1[0.5]$ \\
\hline \multirow[t]{7}{*}{ Arp $295 \ldots \ldots \ldots \ldots$} & Arp 295a & $19.74 \pm 0.19$ & $1.04 \pm 0.21$ & $5.32 \pm 0.09 *$ & $8.6 \pm 0.1$ & $10.1 \pm 0.1$ & $9.4[8.2]$ & $3.2[0.2]$ \\
\hline & Arp 295b & $16.96 \pm 0.06$ & $0.39 \pm 0.09$ & $3.03 \pm 0.06^{*}$ & $8.4 \pm 0.1$ & $9.6 \pm 0.1$ & 9.8 [7.9] & $13.1[0.2]$ \\
\hline & Southwest tail & $19.98 \pm 0.21$ & $0.34 \pm 0.27$ & $3.48 \pm 0.18^{*}$ & $8.2_{-1.0}^{+0.3}$ & $8.6_{-0.3}^{+0.1}$ & $9.1[8.1]$ & $1.5[0.2]$ \\
\hline & Bridge & $20.87 \pm 0.58$ & $1.00 \pm 0.65$ & $3.68 \pm 0.28^{*}$ & $8.6_{-0.2}^{+0.1}$ & $8.6 \pm 0.1$ & $8.8[8.3]$ & $0.5[0.2]$ \\
\hline & Plume & $18.87 \pm 0.10$ & $0.12 \pm 0.15$ & $4.53 \pm 0.11^{*}$ & Cont. & $9.1 \pm 0.1$ & $9.8[8.3]$ & $5.8[0.2]$ \\
\hline & Clump 1 & $20.56 \pm 0.15$ & $-0.09 \pm 0.21$ & $1.87 \pm 0.15^{*}$ & $6.5_{-0.2}^{+1.2}$ & $6.8_{-0.1}^{+0.6}$ & $8.8[7.2]$ & $6.4[0.2]$ \\
\hline & Clump 2 & $21.34 \pm 0.20$ & $0.10 \pm 0.23$ & $2.36 \pm 0.16^{*}$ & $7.8_{-1.3}^{+0.5}$ & $7.2_{-0.2}^{+0.1}$ & $8.4[7.1]$ & $3.4[0.2]$ \\
\hline \multirow[t]{8}{*}{ NGC 520} & NGC 520a & $16.83 \pm 0.05$ & $0.78 \pm 0.06$ & $3.77 \pm 0.14^{*}$ & $8.6 \pm 0.1$ & $9.4 \pm 0.1$ & $8.8[6.9]$ & $9.9[0.1]$ \\
\hline & NGC 520b & $19.00 \pm 0.06$ & $0.96 \pm 0.06$ & $4.24 \pm 0.13^{*}$ & $8.6 \pm 0.1$ & $8.9 \pm 0.1$ & $8.1[6.5]$ & $5.0[0.1]$ \\
\hline & NGC 520c & $19.12 \pm 0.07$ & $0.71 \pm 0.08$ & $3.88 \pm 0.14 *$ & $8.5 \pm 0.1$ & $8.5 \pm 0.1$ & $8.3[6.5]$ & $7.9[0.1]$ \\
\hline & UGC 957 & $19.42 \pm 0.09$ & $0.40 \pm 0.13$ & $3.00 \pm 0.16^{*}$ & $8.4 \pm 0.1$ & $7.7 \pm 0.1$ & $8.5[7.1]$ & $3.2[0.1]$ \\
\hline & Southeast tail & $19.28 \pm 0.07$ & $1.16 \pm 0.08$ & $3.62 \pm 0.14 *$ & $8.6 \pm 0.1$ & $8.4 \pm 0.1$ & $8.4[7.1]$ & $3.1[0.2]$ \\
\hline & Northwest tail & $19.32 \pm 0.07$ & $0.63 \pm 0.08$ & $4.01 \pm 0.14 *$ & $8.4 \pm 0.1$ & $8.5 \pm 0.1$ & 8.6 [6.9] & $6.2[0.1]$ \\
\hline & Clump 1 & $19.91 \pm 0.09$ & $0.70 \pm 0.11$ & $3.19 \pm 0.14 *$ & $8.5 \pm 0.1$ & $7.7 \pm 0.1$ & $7.6[6.3]$ & $2.6[0.1]$ \\
\hline & Clump 2 & $20.04 \pm 0.09$ & $1.09 \pm 0.10$ & $4.16 \pm 0.14^{*}$ & $8.6 \pm 0.1$ & $8.4 \pm 0.1$ & $7.9[6.5]$ & $3.0[0.1]$ \\
\hline
\end{tabular}

${ }^{a}$ NUV $-R$ for Arp 295 or NUV $-V$ for NGC 520, as indicated by an asterisk.

${ }^{\mathrm{b}}$ Ages are reported for the case of an instantaneous burst, with the exception of the Plume in Arp 295 (which was best-fitted by continuous star formation models). The colors of NGC 7771b and clump 2 in NGC 5719 were just outside the grid of synthetic predictions (due to observational scatter), so in these cases we report ages based only on FUV - NUV.

${ }^{c}$ The minimum reported $1 \sigma$ error on derived mass is 0.1 dex, which is larger than the smallest photometric error, but consistent with a maximum error of $10 \%$ in assumed distance.

${ }^{\mathrm{d}}$ The numbers in brackets are $\mathrm{H}$ I detection limits, based on a $3 \sigma$ noise/beam in a single channel over the region of interest. All errors are smaller than or equal to $0.1 \mathrm{dex}$, the error in assumed distances.

5713/19, Langston \& Teuben (2001); Arp 295 and NGC 520, Hibbard \& van Gorkom (1996). ${ }^{12}$ Generally, the synthesized beam measured $\sim 30^{\prime \prime}$, with integrations sufficient to accurately map H I column densities greater than a few $\times 10^{19} \mathrm{~cm}^{-2}$.

The $\mathrm{H}$ I distribution is seen to be closely matched to the FUV emission (Fig. 1). The $\mathrm{H}$ I and FUV emission generally peak at the same locations in the tidal tails (within the accuracy of the $\mathrm{H}$ I resolution). The $\mathrm{H}$ I surface density generally exceeds $\sim 2$ $M_{\odot} \mathrm{pc}^{-2}$ where FUV emission is detected (Table 1). In locations where the $\mathrm{H} \mathrm{I}$ is below this level, FUV emission is usually not detected. This suggests that current star formation is directly related to the cold gas density and that a threshold density must be reached before star formation can begin. Assuming that $\mathrm{CO}$ is coincident with $\mathrm{H}$ I suggests that the observed ( $\mathrm{H} \mathrm{I}$ ) density of $\sim 2 M_{\odot} \mathrm{pc}^{-2}$ is a lower limit to the star formation threshold density in tidal tails. Higher resolution $\mathrm{H} \mathrm{I}$ and spatially resolved $\mathrm{CO}$ images would be very helpful in exploring this possibility.

\subsection{Visible-Band Observations}

Photometry at visible wavelengths was performed for the selected UV-bright regions (sky was determined from multiple $15^{\prime \prime} \times 15^{\prime \prime}$ squares; fluxes of individual pixels were summed using IRAF). For NGC 7771 we used optical images in the Sloan Digital Sky Survey (SDSS) gri bands from the Isaac Newton Telescope ${ }^{13}$ Wide Angle Survey (McMahon et al. 1999), and for NGC 5719 we used SDSS ugriz images. Optical

\footnotetext{
${ }^{12}$ Thanks to T. Nordgren, G. Langston, and J. Hibbard for sharing their data.

${ }^{13}$ The Isaac Newton Telescope is operated on the island of La Palma by the Isaac Newton Group in the Spanish Observatorio del Roque de los Muchachos of the Instituto de Astrofísica de Canarias.
}

images for Arp $295(B V R)$ and NGC $520(V)$ were obtained by Hibbard \& van Gorkom (1996). ${ }^{14}$ After removal of field stars, we computed apparent magnitudes with associated errors, including calibration and background subtraction uncertainties (but excluding photon noise). In cases in which no optical emission was detected, we determined $1 \sigma$ upper limits.

There are many locations where UV emission and $\mathrm{H}$ I are detected but no optical emission is evident (Fig. 1); for example, NGC 7771b, the extended southeast tidal tail of NGC 5713, or the plume east of Arp 295b. In these regions, the H I column density is always $\gtrsim 2 M_{\odot} \mathrm{pc}^{-2}$. In a few regions, optical light is detected with no corresponding UV or $\mathrm{H} \mathrm{I}$; for example, at the end of the southwest tail or in the center of the bridge in Arp 295, or from the red plume extending north from NGC 520. In these regions, the colors are indicative of old stars presumably pulled from the progenitor galaxies during the interaction.

\section{INTERPRETATION}

\subsection{Photometric Ages}

Luminosity-weighted average ages and extinction for the stellar populations in UV-bright regions were estimated by comparing observed FUV - NUV and NUV - optical colors with reddened synthetic predictions (Bruzual \& Charlot 2003 models, computed as described by Bianchi et al. 2004). The observed colors were corrected for foreground extinction (Schlegel et al. 1998) prior to model fitting. The inclusion of the UV-optical color was vital, as it discounted the possibility that any of the observed FUV - NUV colors were due to hot evolved main-

\footnotetext{
${ }^{14}$ Thanks to J. Hibbard for making the calibrated data available.
} 
sequence stars with ages $\gtrsim 10^{9.9}$ yr. In Table 1 we give ages derived under the assumption of (single) instantaneous burst star formation. These values represent lower limits to the actual ages (the integrated color of a mixed-age population is made bluer by the most recent burst, unless the mass in stars formed is insignificant relative to the preexisting total). We emphasize that a good match to the synthetic colors of a recent burst does not preclude star formation at earlier times, except in the limit where the latest burst fully accounts for the bolometric luminosity of a source. The Arp 295 plume was the only source having colors better fitted by the alternate case of continuous star formation. In this scenario, synthetic colors remain blue indefinitely. It is plausible that the plume has hosted recurrent bursts (of which clumps 1 and 2 are the most recent) occurring at a rate that makes star formation in the region appear pseudocontinuous. For all UV-selected regions, we also estimated stellar masses (Table 1) based on the extinction-corrected FUV magnitude and the best matching synthetic model.

The (FUV - NUV, NUV - optical) ages for UV-selected regions cover a substantial range. NGC 7771 and NGC 5719, in the earliest interactions, have UV-bright regions ranging from less than 3 Myr (NGC 7771b, NGC 5719 clumps) to 400 Myr. Arp 295 exhibits very recent star formation only in the eastern plume $(<8 \mathrm{Myr}$ ), while the rest of the system appears to have ages between 80 and 400 Myr. The NGC 520 system (oldest interaction) shows no evidence for current star formation, and the youngest regions are 300 Myr (in UGC 957). Since the UV-bright regions identified by GALEX are considerably younger than the dynamical ages of the tails ( $2500 \mathrm{Myr}$, based on morphology and models; Mihos \& Hernquist 1994, 1996), the bursts of star formation must occur during the evolution of the tidal tails. It would be invaluable to have reliable determinations of dynamic histories of all of these systems (e.g., through targeted models).

\subsection{Star Formation History in the Tails}

The UV observations allow us to begin tracing the star formation history of the tidal tails. In NGC 7771, the youngest stars are at the end of the H I tail. In NGC 5719, the youngest clump is furthest from the galaxy body; the diffuse southeast tail is considerably younger than the northwest tail (which is younger than the galaxy disk). In Arp 295, the clumps in the plume are the youngest, followed by the plume itself; the long tail that has been ejected from Arp 295a is younger at its southwest extreme and older in the inner regions (the bridge). In NGC 520, the youngest stars are in UGC 957. We also find ( $\$ 2.3$ ) that the brightest UV emission coincides with $\mathrm{H}$ I peaks; examination of Table 1 shows that these locations are also the bluest (youngest). Star formation is apparently still occurring in the possible TDGs, but not in the knots closer to the parent galaxy. This could be because the critical density takes longer to develop as the distance from the parent galaxy increases, or because star formation has been inhibited at the tail/galaxy interface.

One question of great interest is whether or not the starforming clumps in the tidal tails are destined to remain as independent entities- that is, as TDGs. Of the compact UVbright regions well separated from the primary interacting galaxies, several of our sources have $\mathrm{H}$ I mass comparable to confirmed TDGs. Duc \& Mirabel (1998) indicate TDGs have H I mass ranging from $10^{8.3}$ to $10^{9.8} M_{\odot}$. Within our own sample, TDG UGC 957 (near NGC 520) has $10^{8.5} M_{\odot}$ of H I, and the TDGs in NGC $4038 / 39$ have $10^{8.2}-10^{8.4} M_{\odot}$ of H I (Hibbard et al. 2001). In comparison, NGC 7771b, Arp 295 clump 1, and Arp 295 clump 2 have $10^{8.7}, 10^{8.8}$, and $10^{8.4} M_{\odot}$, respectively, of $\mathrm{H}$ I coincident with UV emission, making them plausible TDG candidates. High-resolution $21 \mathrm{~cm}$ observations that are able to gauge the velocity dispersion within NGC 7771b, Arp 295 clump 1, and Arp 295 clump 2 (and even slightly lower mass condensations like NGC 5719 clumps 1 and $2,10^{7.5}-10^{7.8} M_{\odot}$ of $\mathrm{H}$ I) are needed to determine conclusively if they are self-gravitating. Regardless, our early GALEX observations demonstrate the effectiveness of vacuum UV imaging as a means of identifying TDG candidates, particularly at the lower limit of the mass function.

GALEX is a NASA small explorer launched in 2003 April. We gratefully acknowledge NASA's support for construction, operation, and science analysis for the GALEX mission, developed in cooperation with the Centre National d'Etudes Spatiales of France and the Korean Ministry of Science and Technology

\section{REFERENCES}

Barnes, J. E., \& Hernquist, L. E. 1992, ARA\&A, 30, 705

Braine, J., Duc, P.-A., Lisenfeld, U., Charmandaris, V., Vallejo, O., Leon, S., \& Brinks, E. 2001, A\&A, 378, 51

Bruzual, G., \& Charlot, S. 2003, MNRAS, 344, 1000

de Grijs, R., Lee, J. T., Clemencia Mora Herrera, M., Fritze-v. Alvensleben, U., \& Anders, P. 2003, NewA, 8, 155

Duc, P.-A., \& Mirabel, I. F. 1998, A\&A, 333, 813

Haynes, M. P., \& Giovanelli, R. 1991, ApJS, 77, 331

Hibbard, J. E., Vacca, W. D., \& Yun, M. 2000, AJ, 119, 1130

Hibbard, J. E., \& van Gorkom, J. H. 1996, AJ, 111, 655

Hibbard, J. E., van Gorkon, J. H., Rupen, M. P., \& Schiminovich, D. 2001, in ASP Conf. Ser. 240, Gas and Galaxy Evolution, ed. J. E. Hibbard, M. P. Rupen, \& J. H. van Gorkom (San Francisco: ASP), 659

Hibbard, J. E., et al. 2005, ApJ, 619, L87

Knierman, K. A., Gallagher, S. C., Charlton, J. C., Hunsberger, S. D., Whitmore, B., Kundu, A., Hibbard, J. E., \& Zaritsky, D. 2003, AJ, 126, 1227
Kotilainen, J. K., Reunanen, J., Laine, S., \& Ryder, S. D. 2001, A\&A, 366, 439 Langston, G., \& Teuben, P. 2001, in ASP Conf. Ser. 240, Gas and Galaxy Evolution, ed. J. E. Hibbard, M. P. Rupen, \& J. H. van Gorkom (San Francisco: ASP), 862 Martin, D. C., et al. 2005, ApJ, 619, L1

McMahon, R. G., Walton, N. A., Irwin, M. J., Lewis, J. R., Bunclark, P. S., \& Jones, D. H. 2001, NewA Rev., 45, 97

Mihos, J. C., \& Hernquist, L. E. 1994, ApJ, 427, 112 1996, ApJ, 464, 641

Mirabel, I. F., Dottori, H., \& Lutz, D. 1992, A\&A, 256, L19

Nordgren, T. E., Chengalur, J. M., Salpeter, E. E., \& Terzian, Y. 1997, AJ, 114, 913

Saviane, I., Hibbard, J. E., \& Rich, R. M. 2004, AJ, 127, 660

Schlegel, D. J., Finkbeiner, D. P., \& Davis, M. 1998, ApJ, 500, 525

Schombert, J. M., Wallin, J. F., \& Struck-Marcell, C. 1990, AJ, 99, 497

Stanford, S. A., \& Balcells, M. 1990, ApJ, 355, 59 